\title{
CONDILOMAS ANO-GENITAIS EXUBERANTES: ABORDAGEM CLÍNICA E TERAPÊUTICA
}

\author{
Ana Maria Calistru', Paulo Santos², Cármen Lisboa ${ }^{3 *}$, Filomena Azevedo 4 \\ IInterna do Internato Complementar de Dermatologia e Venereologia/Resident, Dermatology and Venereology \\ ${ }^{2}$ Assistente Hospitalar Graduado de Dermatologia e Venereologia/Graduated Consultant of Dermatology and \\ Venereology \\ ${ }^{3}$ Professora Doutora, Especialista em Dermatologia e Venereologia/Professor, Consultant of Dermatology and \\ Venereology \\ ${ }^{4}$ Directora de Serviço de Dermatologia e Venereologia/Head, Dermatology and Venereology Department \\ Serviço de Dermatologia e Venereologia, Hospital de S. João EPE, Porto, Portugal \\ *Faculdade de Medicina da Universidade do Porto, Portugal
}

RESUMO - A abordagem dos condilomas acuminados ano-genitais exuberantes (CAE) constitui um desafio. As opções terapêuticas são limitadas, a taxa de recorrência é alta, associam-se com imunossupressão e tem maior risco de transformação maligna. Analisamos os processos clínicos de 53 doentes observados por CAE (área total da lesão $>5 \mathrm{~cm}^{2}$ ) na nossa unidade de Infecções Sexualmente Transmissíveis por um período de 10 anos (2000-2009). Foram incluídos 50 homens e 3 mulheres, com idade média de 39,9 anos, a maioria $(n=47,88,6 \%)$ heterossexuais, tendo a infecção pelo vírus da imunodeficiência humana $(\mathrm{VIH}) 21$ doentes $(39,6 \%)$. Observamos condiloma gigante de Buschke-Lowenstein em 33,3\% dos doentes $\mathrm{VIH}+$, comparativamente com 6,2\% dos $\mathrm{VIH}$-. Foram utilizados vários métodos terapêuticos, em monoterapia ou em combinação: criocirurgia $(n=44)$, electrocirurgia $(n=9)$, laser $\mathrm{CO} 2$ $(n=2)$ e exérese $(n=3)$, com uma média de 4,7 sessões para os doentes $\mathrm{VIH}+$ e 2,6 para os restantes. Tiveram resolução clínica 31 doentes (sem evidência de lesões após 6 meses) e 22 doentes tiveram melhoria inicial mas abandonaram o seguimento. Registou-se reaparecimento de lesões, embora de menores dimensões, em $69 \%$ dos doentes $\mathrm{VIH}+$ e 38\% dos $\mathrm{VIH}$-. A baixa adesão dos doentes acrescenta-se aos problemas encontrados no tratamento dos CAE, $41 \%$ dos nossos doentes tendo abandonado o seguimento. Consideramos a crioterapia eficaz na redução do volume dos $\mathrm{CAE}$, permitindo a resolução após sessões subsequentes do mesmo ou outro método cirúrgico.

PALAVRAS-CHAVE - Papilomavirus Humano (HPV); Condiloma Acuminado; Ano-genital; Tumor de Buschke-Lowenstein; Vírus da Imunodeficiência Humana (VIH).

\section{LARGE ANO-GENITAL WARTS: CLINICAL FEATURES AND MANAGEMENT}

ABSTRACT - The management of the large anogenital warts (LAW) is a challenge due to limited treatment options, high recurrence rates, association with immunosuppression and the risk of malignant transformation. We analyzed the clinical records of 53 patients with LAW (total wart area more than $5 \mathrm{~cm} 2$ ) attended in our Sexual Transmitted Infections Unit during 10 years (2000-2009). The study included 50 men and 3 women, with a mean age of 39,9 years (range 21-74). The majority ( $n=47,88,6 \%)$ was heterosexual . and 21 patients $(39,6 \%)$ had the human immunodeficiency virus infection (HIV). Buschke-Lowenstein giant condyloma was seen in 33,3\% of the HIV+ patients comparing with 6,2\% of the HIV-patients. Different therapeutic methods, as monotherapy or in combination, were used: cryosurgery $(n=44)$, electrosurgery $(n=9)$, CO2 laser ( $n=2)$ and excision ( $n=3)$, with a mean number of 4,7 sessions in the HIV+ patients and 2,6 in the HIV-patients. Clinical clearance patients achieved in 31 patients (no lesions and no evidence of disease at 6 months follow-up) and 22 patients showed initial improvement but were lost at follow-up. Recurrence of the lesions, although of lesser extent, was seen after a mean interval of 2,7 years in $69 \%$ of the HIV+ patients and in $38 \%$ of the HIV-patients. The patient low compliance adds to the problems encountered in the treatment of LAW, as $41 \%$ of the patients abandoned the follow-up. Cryotherapy is effective in the lesion's volume reduction, allowing further clearance with the same or another surgical method. 


\section{GEIDST}

KEY-WORDS - Human Papillomavirus (HPV); Condyloma Acuminatum, Anogenital; Buschke-Lowenstein Tumor; Human Immunodeficiency Virus (HIV).

Conflitos de interesse: Os autores declaram não possuir conflitos de interesse. No conflicts of interest.

Dr.. Ana Maria Calistru

Serviço de Dermatologia e Venereologia

Hospital de São João EPE, Porto

Alameda Professor Hernâni Monteiro

4200-319, Porto, Portugal

Tel. / Fax: 00351225512193

Telemóvel: 00351960147387

E-mail: anaguia@yahoo.com

\section{INTRODUÇÃO}

A infecção genital por papilomavírus humano (HPV) é muito comum, estimando-se em cerca de 15\% da população geral ${ }^{1}$. Pode ser assintomática, causar neoplasia intraepitelial ou carcinoma invasivo, ou pode manifestar-se por condilomas ano-genitais que afectam $1 \%$ da população geral ${ }^{2}$. Os condilomas acuminados ano-genitais exuberantes (CAE) representam uma minoria das infecções causadas por HPV, mas a abordagem deles constitui um desafio devido a vários factores: opções terapêuticas limitadas, alta taxa de recorrência, associação com a imunoSsupressão e maior risco de transformação maligna.

\section{ESTUDO CLÍNICO}

O presente trabalho tem como objectivo caracterizar a população com CAE (área total da lesão $>5 \mathrm{~cm}^{2}$ ) e estabelecer a eficácia e tolerância da crioterapia e de outros métodos cirúrgicos. Do total de 637 de doentes observados por condilomas acuminados na nossa Unidade de Infecções Sexualmente Transmissíveis (IST) num período de 10 anos (2000-2009), foram analisados os processos clínicos de 53 doentes (8,3\%) que tinham CAE. Foram incluídos os dados sobre sexo, idade, comportamento sexual, aspectos clínicos das lesões, tipo de tratamento e número de sessões realizadas, resultados e efeitos secundários. Ainda avaliamos a associação de outras ISTs e a contagem de células CD4+ nos doentes com infecção pelo vírus da imunodeficiência humana (VIH). Utilizamos o programa SPSS 19 na analise estatística dos dados, considerando-se uma diferença significativa para um valor do $p<0,05$.

Foram incluídos 50 homens e 3 mulheres, todos caucasianos, com idade compreendida entre 21 e 74 anos, a média sendo de 39,9 anos. A maioria dos doentes $(\mathrm{n}=47,88,6 \%)$ referiam ser heterossexuais e 6 homens tinham sexo com homens. Dezasseis (30\%) doentes tiveram 2 ou mais parceiros sexuais nos últimos 6 meses. No caso dos homens as lesões estavam localizadas no pénis $(n=19)$, região perianal $(n=15)$, região inguinal $(n=1)$ (Fig. 1) ou concomitantemente no pénis e região perianal $(n=13)$ ou no pénis e virilhas $(n=2)$. Dois homens tinham ainda condilomas acuminados da mucosa oral. Nas mulheres as lesões localizavam-se na vulva e região perianal $(n=2)$ ou apenas na vulva $(n=1)$. Duas das três mulheres tinham infecção por HPV no colo uterino. A duração das lesões variou entre 2 meses e 4 anos. Constatou-se coexistência de outras ISTs em 25 doentes, nomeadamente: infecção por VIH $(n=21)$, hepatite $C(n=7)$, sífilis latente $(n=3)$, hepatite B crónica $(n=1)$, herpes genital $(n=1)$, uretrite por Chlamydia trachomatis $(n=1)$ e molusco contagioso $(n=1)$.

Observamos a forma de condiloma gigante de Buschke-Lowenstein (Figura 2) em 7 doentes VIH+ (correspondendo a $33,3 \%$ ) e em 2 doentes $\mathrm{VIH}$ - (6,2\%), esta 


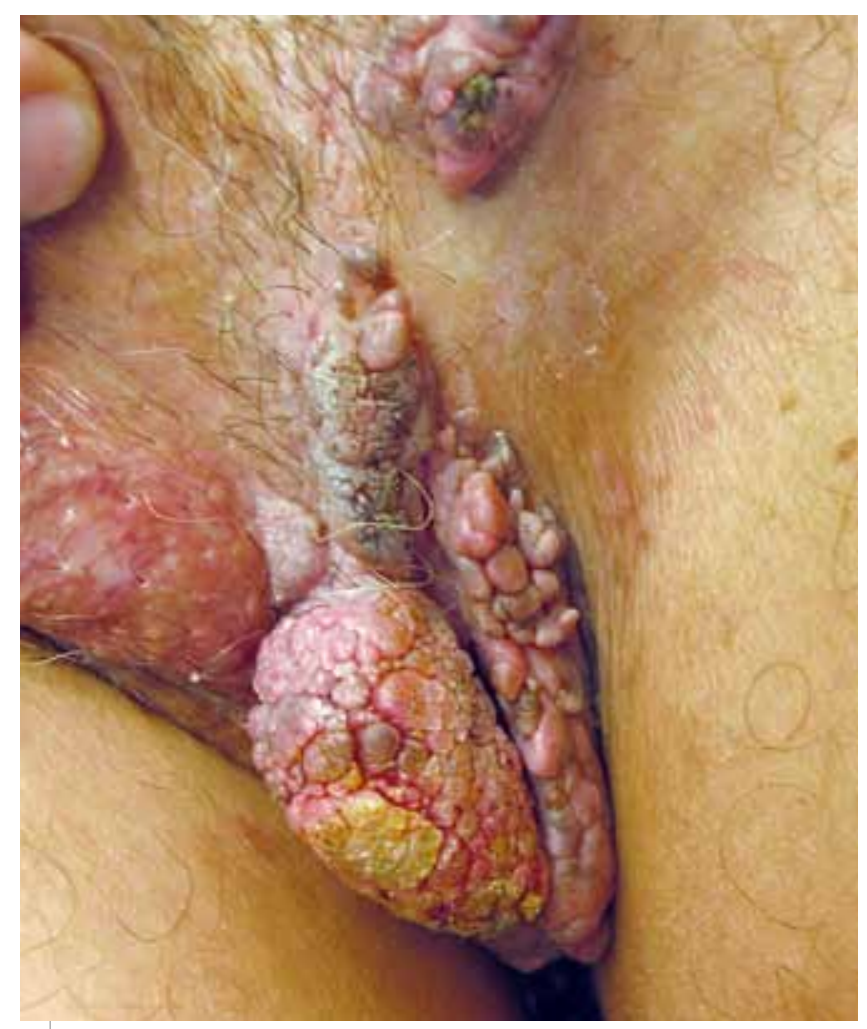

Fig. 1 - Condiloma de grandes dimensões na região inguinal.

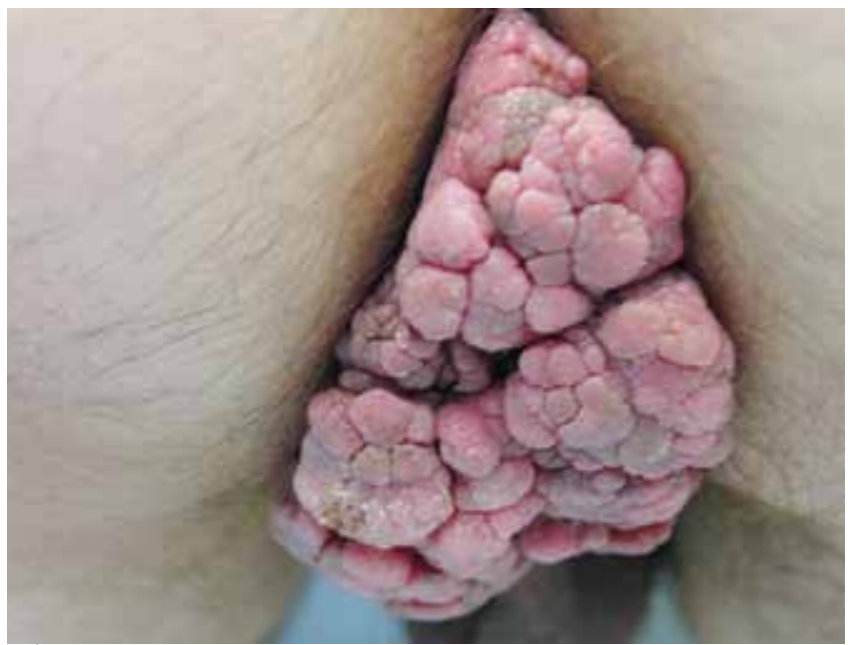

Fig. 2 - Tumor de Buschke-Lowenstein perianal.

diferença sendo estatisticamente significativa $(p=0,01)$. Eram todos homens, sendo a localização perianal em 7 pacientes e peniana nos outros 2 . Foi realizada biópsia em 4 casos, com evidência de neoplasia intraepitelial anal num doente $\mathrm{VIH}+$.
A abordagem terapêutica consistiu em diversos métodos cirúrgicos em monoterapia ou em combinação (Tabela 1), sendo necessárias várias sessões por cada doente. A criocirurgia foi o método mais utilizado e consistindo em congelação da lesão com duração de 15-60 segundos por ciclo (nalguns casos sendo necessários tempos superiores), com 2-3 ciclos por sessão (Fig. 3). A criocirurgia foi realizada em monoterapia em 39 doentes e em combinação sequencial com laser $\mathrm{CO} 2$, electrocirurgia ou exérese cirúrgica em 5 doentes. A electrocirurgia em monoterapia foi utilizada em 7 doentes e a exérese cirúrgica isolada em 2 doentes. Os doentes com lesões da região perianal foram avaliados em consulta de Gastroenterologia-Proctologia e 9 deles foram tratados com laser árgon por envolvimento do canal anal e/ou recto. O número de sessões de tratamento variou entre 1 e 24 por doente, tendo uma média de 4,7 para os doentes $\mathrm{VIH}+$ e de 2,6 para os

Tabela 1 - Métodos terapêuticos utilizados

\begin{tabular}{|c|c|c|}
\hline \multirow{2}{*}{ Método terapêutico } & No doentes \\
\hline \multirow{4}{*}{ Monoterapia } & Criocirurgia & 39 \\
\cline { 2 - 3 } & Electrocirurgia & 7 \\
\cline { 2 - 3 } & Exérese & 2 \\
\hline \multirow{2}{*}{$\begin{array}{c}\text { Terapêutica } \\
\text { combinada* }\end{array}$} & Criocirurgia+Electrocirurgia & 2 \\
\cline { 2 - 3 } & Criocirurgia+Laser CO2 & 2 \\
\cline { 2 - 3 } & Criocirurgia+Exérese & 1 \\
\hline
\end{tabular}

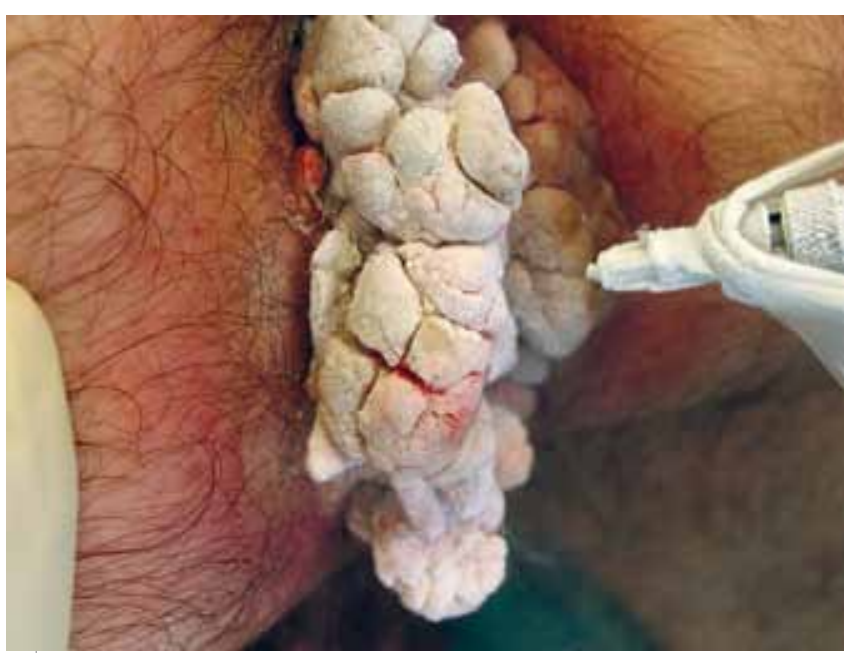

Fig. 3 - Criocirurgia de condiloma exuberante perianal. 


\section{GEIDST}

restantes. A recuperação pós-operatória demorou entre 2 a 6 semanas. As complicações mais frequentes foram a dor, a ulceração e as alterações de pigmentação enquanto que a hemorragia, a infecção, e a disfunção do esfíncter anal constituíram complicações raras. Trinta e um doentes tiveram resolução clínica e encontravam-se sem evidência das lesões após 6 meses e 22 doentes tiveram melhoria inicial mas abandonaram o seguimento (Tabela 2). Entre os 31 doentes com resolução clínica registou-se reaparecimento dos condilomas, embora de menores dimensões, em 16 doentes $(51,6 \%$ dos casos), após um intervalo médio de 2,7 anos, sendo a taxa de recorrência de $69 \%$ nos doentes $\mathrm{VIH}+$, e de $38 \%$ nos doentes VIH- $(p=0,09)$. No que respeita ao número dos linfócitos $\mathrm{T}$ CD4 + nos doentes $\mathrm{VIH}+$, não encontramos diferença estatisticamente significativa entre o grupo com menos de $200 / \mathrm{mmc}$ e o grupo com mais de $200 / \mathrm{mmc}$, relativamente ao número de lesões de tipo Buschke-Lowenstein (4 e 5 casos, respectivamente, $p=0,2$ ) ou ao número de recidivas ( 4 e 3 casos, respectivamente, $p=0,6)$.

Tabela 2 - Resultado do tratamento comparando os subgrupos de doentes $\mathrm{VIH}$ - e $\mathrm{VIH}+$

\begin{tabular}{|c|c|c|c|c|}
\hline & \multicolumn{2}{|c|}{ Resolução clínica } & \multirow[b]{2}{*}{ Abandono** } & \multirow[b]{2}{*}{ Total } \\
\hline & $\underset{\text { recidiva* }}{\text { Sem }}$ & $\underset{\text { recidiva* }}{\text { Com }}$ & & \\
\hline VIH- & 11 & 7 & 14 & 32 \\
\hline VIH+ & 4 & 9 & 8 & 21 \\
\hline Total & 15 & 16 & 22 & 53 \\
\hline
\end{tabular}

\section{DISCUSSÃO}

A maioria dos doentes do nosso estudo foi constituída por homens heterossexuais, sendo as lesões localizadas predominantemente no pénis e região perianal.

Observamos uma proporção importante de doentes $\mathrm{VIH}+(39,6 \%)$ na população com CAE e ainda um risco significativo de desenvolver a forma de condiloma gigante de Buschke-Lowenstein neste grupo. Nos doentes $\mathrm{VIH}+$, devido ao défice da imunidade celular, a infecção por HPV é mais frequente, tem a tendência de persistir e de progredir para neoplasias intraepiteliais, sendo factores importantes a depleção de células $\mathrm{T} C D 4+$ e a carga viral $\mathrm{VIH}^{3}$. A tendência para persistência do HPV nos doentes VIH+ pode explicar a necessidade de um maior número de tratamentos e a maior taxa de recidiva confirmados pelo presente trabalho. No entanto, não notamos aumento significativo do risco de lesões tipo Buschke-Lowenstein ou de recidivas nos doentes com número de células T CD4+ menor de $200 / \mathrm{mmc}$, provavelmente devido ao número reduzido de casos.

Os condilomas ano-genitais são causados habitualmente pelos tipos 6 e 11 do HPV, considerados de baixo risco oncogénico. Contudo, as lesões grandes, infiltrativas de tipo Buschke-Lowenstein tem potencial invasivo e destrutivo local, e raramente, podem originar metástases. Além disso, a co-infecão com tipos de alto risco é detectável num terço dos condilomas genitais ${ }^{4}$, sendo importante a bióspia nas lesões grandes, infiltrativas, ou na papulose bowenoide bem como perante a resistência ao tratamento.

A terapêutica dos condilomas ano-genitais tem como objectivo a resolução clínica e a remissão duradoura, não havendo método que elimine completamente o vírus ${ }^{4}$. As opções terapêuticas incluem métodos cirúrgicos/destrutivos (criocirurgia, electrocirurgia, laser, exérese cirúrgica, aplicação de ácido tricloroacético 70-90\%), tratamentos citotóxicos ou antivíricos (podofilotoxina tópica, cidofovir tópico ou intralesional) e imunomoduladores (imiquimod tópico, interferão intralesional ou sistémico). Apenas os métodos cirúrgicos tem taxa de resolução clínica primária perto de $100 \%$, e as recorrência podem ocorrer após qualquer tipo de tratamento, sendo de pelo menos $20-30 \%{ }^{5}$. A crioterapia em condilomas não seleccionados tem uma taxa de resolução clínica de $44-75 \%$ e uma recorrência de $21-42 \%{ }^{6-8}$. Nos doentes com CAE observamos - reaparecimento de condilomas numa proporção significativa dos casos (53\%), provavelmente devido a maior dimensão da lesão inicial, a associação com a imunossupressão ou a reinfecção. A baixa adesão dos doentes acrescenta-se aos problemas encontrados no tratamento dos CAE, $41 \%$ dos nossos doentes tendo abandonado a consulta antes do fim de período de seguimento de 6 meses. Consideramos a crioterapia eficaz na redução do volume dos $C A E$, permitindo a resolução após sessões subsequentes do mesmo ou outro método cirúrgico.

Vários estudos mostraram a eficácia da vacinação HPV quadrivalente na prevenção das infecções com as respectivas estirpes e das lesões pre-neoplásicas ${ }^{9}$. No futuro, a vacinação terapêutica poderá trazer benefícios no controlo desta infecção, sobretudo em grupos de risco, como os doentes $\mathrm{VIH}+$. 


\section{REFERÊNCIAS}

1. Scheinfeld N, Lehman DS. An evidence-based review of medical and surgical treatments of genital warts. Dermatol Online J.2006;12(3):5.

2. Mayeaux EJ, Dunton C. Modern management of external genital warts. J Low Genit Tract Dis. 2008; 12(3):185-92.

3. Kirnbauer R, Lenz P, Okun M. Human Papillomavirus. In: Bolognia J, Jorizzo J, Rapini R, editors. Dermatology, 2nd ed. Espanha. Mosby Elsevier; 2008. 1183-1199.

4. Krogh G. Management of anogenital warts (condylomata acuminata). European Journal of Dermatology. $2001 ; 11(6): 598-604$.

5. Lacey CJN, Woodhall SC, Wikstrom A, Ross J. IUSTI 2010 European Guidelines for the Management of Anogenital Warts in Adults.
6. Stone KM, Becker TM, Hadgu A, Kraus SJ. Treatment of external genital warts: a randomized clinical trial comparing podophyllin, cryotherapy, and electrodesiccation. Genitourin Med.1990;66(1):16-9.

7. Handley JM, Maw RD, Horner T, Lawther H, McNeill T, Dinsmore WW. Non-specific immunity in patients with primary anogenital warts treated with interferon alpha plus cryotherapy or cryotherapy alone. Acta Derm Venereol.1992;72(1):39-40.

8. Sherrard J, Riddell L. Comparison of the effectiveness of commonly used clinic-based treatments for external genital warts. Int J STD AIDS.2007; 18(6):365-8.

9. Pomfret TC, Gagnon JM Jr, Gilchrist AT. Quadrivalent human papillomavirus (HPV) vaccine: a review of safety, efficacy, and pharmacoeconomics. J Clin Pharm Ther.2011;36(1):1-9. 